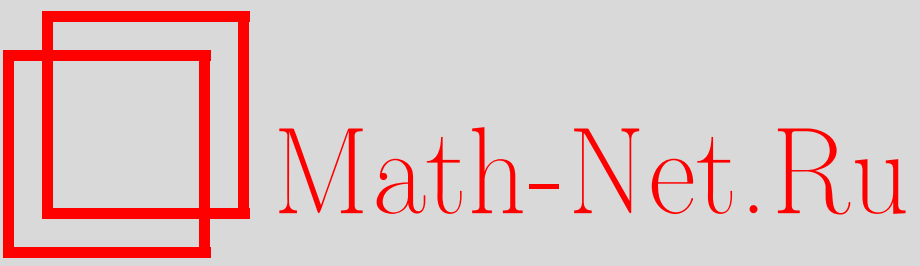

В. П. Радченко, Д. А. Заусаев, Использование банка данных координат больших планет для численного интегрирования уравнений движения небесных тел, Вестн. Сам. гос. техн. ун-та. Сер. Физ.-мат. науки, 2011, выпуск 3(), 202-207

DOI: https://doi.org/10.14498/vsgtu983

Использование Общероссийского математического портала Math-Net.Ru подразумевает, что вы прочитали и согласны с пользовательским соглашением http://www . mathnet.ru/rus/agreement

Параметры загрузки:

IP: 52.23 .180 .231

26 апреля 2023 г., $15: 20: 28$ 
УДК 521.182

\section{ИСПОЛЬЗОВАНИЕ БАНКА ДАННЫХ КООРДИНАТ БОЛЬШИХ ПЛАНЕТ ДЛЯ ЧИСЛЕННОГО ИНТЕГРИРОВАНИЯ УРАВНЕНИЙ ДВИЖЕНИЯ НЕБЕСНЫХ ТЕЛ}

\section{В. П. Радченко, Д. А. Заусаев}

Самарский государственный технический университет,

443100, Самара, ул. Молодогвардейская, 244.

E-mails: radch@samgtu.ru, zadmitriy@gmail.com

Созданы банки данных координат болъших планет на основе численной теории движения планет DE405. Проведено численное интегрирование уравнений движения малых тел Солнечной системы с использованием полученных банков данных. Показана высокая эффективность использования банков данных координат больших планет при численном интегрировании уравнений движения небесных meл.

Ключевые слова: интерполяция, астероидь, кометъ, численное интегрирование.

В настоящее время накоплен большой объём информации о населенности Солнечной системы малыми телами. Благодаря появлению новых наблюдательных технологий произошло существенное увеличение точности определения положений небесных тел. Созданы высокоточные численные теории движения больших планет. Все это позволяет с большей вероятностью реально оценить астероидно-кометную опасность. При решении задач, связанных с проблемой астероидной опасности, требуется проводить регулярные исследования движения большого числа объектов на интервале времени порядка нескольких столетий. Разработка высокоэффективных алгоритмов и программ численного интегрирования уравнений движения небесных тел является необходимым условием для своевременного получения результатов проведенных исследований.

Численное решение уравнений движения небесного тела можно проводить либо путем совместного интегрирования возмущаемого тела и возмущающих планет, либо путем решения уравнений движения возмущаемого тела, при этом координаты планет извлекаются из заранее созданного банка данных. Если координаты и скорости больших планет задаются табличным массивом с определённым интервалом, то внутри интервала их координаты и скорости на нужный момент времени можно получать с помощью различных интерполяционных формул.

В работе [1] рассмотрен способ хранения положений больших планет не в виде координат и скоростей, заданных с определённым интервалом времени, а в форме оскулирующих элементов орбит с шагом один и десять дней. Внутри каждого промежутка координаты и скорости для каждой планеты (Меркурий - Плутон) вычислялись по формулам невозмущенного движения с помощью оскулирующих эллипсов.

Целью данной работы являлось упрощение алгоритма интерполяции, приводящее к ускорению процесса интегрирования уравнений движения небесных тел. Для

Владимир Павлович Радченко (д.ф.-м.н., проф.), зав. кафедрой, каф. прикладной математики и информатики. Дмитрий Анатольевич Заусаев, аспирант, каф. прикладной математики и информатики. 
решения поставленной задачи использовалось два вида интерполяции. Обе интерполяции основаны на использовании разложения координат и скоростей численной теории движения планет, Луны и Солнца DE405 во временные ряды $[2,3]$. При этом коэффициенты этих рядов вычислялись с помощью программы численного интегрирования методом Эверхарта. Выражения для определения координат и скоростей методом Эверхарта вычисляются по следующим формулам:

$$
\begin{gathered}
x=x_{1}+\dot{x_{1}} t+F_{1} \frac{t^{2}}{2}+A_{1} \frac{t^{3}}{6}+\cdots+A_{1} \frac{t^{n+1}}{(n+1)(n+2)}, \\
\dot{x}=\dot{x}_{1}+F_{1} t+A_{1} \frac{t^{2}}{2}+A_{2} \frac{t^{3}}{3}+\cdots+A_{1} \frac{t^{n+1}}{(n+1)} .
\end{gathered}
$$

Полиномы $(1),(2)$ не являются рядами Тейлора, а коэффициенты $A_{i}$ вычисляются из условия наилучшего приближения $x$ и $\dot{x}$ с помощью конечных разложений $(1)$, $(2)$.

В настоящее время одной из наиболее точных численных теорий движения больших планет является численная теория DE405. Банк данных DE405 представляет собой данные на внешнем носителе в виде последовательности групп коэффициентов Чебышева, с помощью которых вычисляются координаты и скорости больших планет, Луны и Солнца на заданный момент времени. Каждая группа охватывает интервал времени в 32 дня.

Непосредственное использование банка данных DE405 в программе численного интегрирования уравнений движения небесных тел затруднительно, поскольку координаты и скорости для Меркурия, Луны и больших планет вычисляются не по единому алгоритму. Кроме того, коэффициенты полинома Чебышева в DE405 приведены для барицентра «Земля + Луна», а не для каждого объекта в отдельности. При исследовании движения объектов, проходящих через сферу действия Земли, необходимо учитывать влияние возмущений, находить сближение объекта с Землёй и Луной в отдельности.

При использовании банка данных DE405 создан банк данных барицентрических координат и скоростей больших планет, Луны и Солнца с шагом 1 день на интервале времени с 1600 по 2200 гг. На концах каждого отрезка длиною 1 день получены координаты и скорости указанных выше объектов, полностью совпадающие с DE405. Затем путём численного интегрирования методом Эверхарта были найдены координаты и скорости больших планет, Луны и Солнца в середине каждого интервала. Таким образом, банк данных состоял из набора координат и скоростей больших планет, Луны и Солнца, заданных в начале, середине и конце однодневного интервала.

Для получения координат и скоростей внутри единичного интервала использовались формулы квадратичной интерполяции. Рассмотрим алгоритм интерполяции на примере вычисления координат внутри единичного интервала для отдельного объекта.

Пусть $x_{0}, x_{1}, x_{2}$ - координаты объекта на моменты времени 0; 0,5 и 1 дня. Для вычисления $x(t)$ при $t \in(0 ; 0,5)$ использовалась формула

$$
x(t)=x_{0}+x_{1 / 2}^{1} t+x_{1}^{2} \frac{t(t-1)}{2},
$$

а при $t \in(0,5 ; 1)-$

$$
x(t)=x_{0}+x_{3 / 2}^{1} t+x_{1}^{2} \frac{t(t-1)}{2},
$$

где $x_{1 / 2}^{1}=x_{0}+x_{1}, x_{1}^{2}=x_{3 / 2}^{1}+x_{1 / 2}^{1}, x_{3 / 2}^{1}=x_{2}+x_{1}$.

Использование формул (3), (4) позволяет получать координаты и скорости планет и Луны с высокой степенью точности, так как максимальная погрешность в координатах Луны не превышает $2 \cdot 10^{-6}$ а. е., в координатах Меркурия погрешность 
составляет $1 \cdot 10^{-7}$ а. е. Однако недостатком данного алгоритма интерполяции является большой объём полученного банка данных, который составляет 221 МБ.

С целю уменьшения объёма банка данных для нахождения координат и скоростей больших планет, Луны и Солнца был предложен другой способ интерполяции, основанный также на использовании банка данных DE405. C помощью метода Эверхарта 11-го порядка были найдены коэффициенты $A_{1}, A_{2}, A_{3}, A_{4}$ для планет, Луны и Солнца с шагом 10 дней. Банк данных, наряду с координатами, скоростями, ускорениями для планет, Луны и Солнца содержал также коэффициенты $A_{1}, A_{2}, A_{3}, A_{4}$ в начале каждого десятидневного интервала. Интерполяция для получения координат и скоростей внутри десятидневного интервала проводилась с помощью формул (1), (2). Благодаря увеличению длины интервалов, на которые был разбит весь промежуток интегрирования (1600-2200 гг.), объём банка данных составил 44,1 МБ.

Путём сопоставления полученных координат с данными численной теории DE405 найдены максимальные погрешности в координатах и скоростях внутри интервалов для планет, Луны и Солнца.

Величины максимальных ошибок $\Delta r_{\max }=\sqrt{\Delta x^{2}+\Delta y^{2}+\Delta z^{2}}$ внутри интервала 10 дней, а.е.:

\begin{tabular}{|c|c|}
\hline Солнце .... & $1,95 \cdot 10^{-12}$ \\
\hline Меркурий . & $1,33 \cdot 10^{-9}$ \\
\hline Венера ... & $1,76 \cdot 10^{-11}$ \\
\hline Земля . . & $2,10 \cdot 10^{-8}$ \\
\hline Луна . . & $1,67 \cdot 10^{-6}$ \\
\hline Mapc ... & $2,11 \cdot 10^{-12}$ \\
\hline Юпитер & $2,71 \cdot 10^{-12}$ \\
\hline Сатурн . & $7,48 \cdot 10^{-13}$ \\
\hline Уран .. & $1,73 \cdot 10^{-12}$ \\
\hline Нептун & $1,60 \cdot 10^{-12}$ \\
\hline Ілутон & $7,97 \cdot 10^{-12}$ \\
\hline
\end{tabular}

Величины максимальных ошибок $\Delta V_{\max }=\sqrt{\Delta \dot{x}^{2}+\Delta \dot{y}^{2}+\Delta \dot{z}^{2}}$ внутри интервала 10 дней, а.е./день:

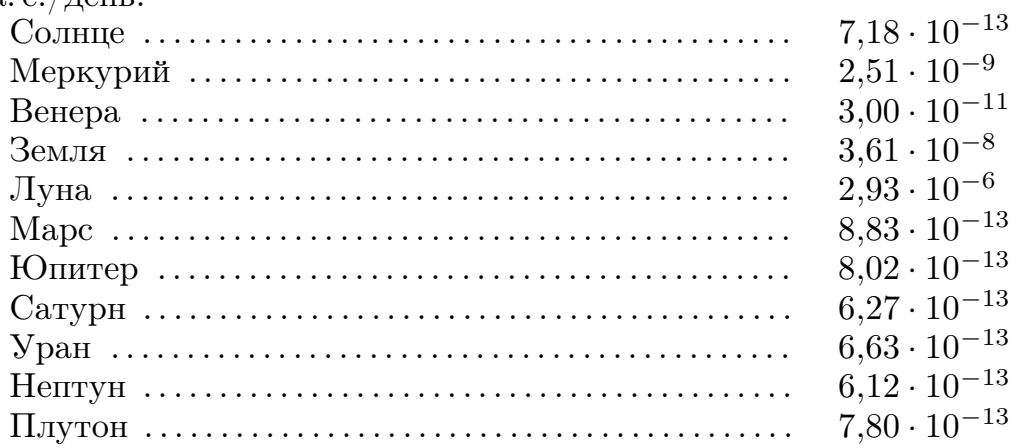

Здесь $\Delta x, \Delta y, \Delta z$ - погрешности в координатах, $\Delta \dot{x}, \Delta \dot{y}, \Delta \dot{z}-$ погрешности в компонентах скоростей.

Из приведённых выше данных видно, что у Луны-наибольшая погрешность в координатах, но вследствие её малой массы по сравнению с большими планетами влияние Луны на движение преобладающего количества небесных тел в Солнечной системе несущественно.

Для определения области применимости данного алгоритма исследовалась эволюция орбит пяти короткопериодических комет и пяти астероидов, принадлежащих к группам Аполлона и Атона. Элементы орбит комет и астероидов взяты из каталогов $[4,5]$, причём все десять комет взяты из начала списка каталога [4], в то время как пять астероидов находятся в конце каталога [5]. Выбор комет, имеющих минимальные порядковые номера, обусловлен тем, что эти кометы имеют достаточно 
точные элементы орбит. Выбор астероидов связан с распределением их минимальных расстояний до Земли. Для численного интегрирования уравнений движения использовался метод Эверхарта 27-го порядка с переменным шагом интегрирования.

В табл. 1 и 2 приведены элементы орбит, полученные путём совместного интегрирования, а также вычисленные по результатам численного интегрирования с использованием банка данных координат, скоростей, ускорений и коэффициентов $A_{1}$, $A_{2}, A_{3}, A_{4}$ больших планет, Луны и Солнца. Результаты интегрирования первым и вторым способом обозначены соответственно «Совм. интегр.» и «Б. дан. коэф»; $\Delta S$ - абсолютные значения разности в орбитальных элементах, полученных первым и вторым методами. Начальные данные элементов орбит комет и астероидов взяты из каталогов [4,5].

Как видно из табл. 1, максимальные расхождения в элементах кометных орбит для всех пяти комет в обоих методах на конце интервала интегрирования незначительны. При этом в угловых элементах максимальное расхождение наблюдается в средней аномалии у кометы D/Biela - 0,0044 градуса, а отличия в остальных элементах орбит не превышают точности оптических наблюдений.

В табл. 2 приведены элементы орбит пяти астероидов, принадлежащих к группам Аполлона и Атона. Каждый из этих астероидов в процессе эволюции не проходит через сферу действия планет. Однако имеются умеренные сближения астероидов с большими планетами, что приводит к расхождению полученных результатов. Следует отметить, что полученное расхождение результатов интегрирования двумя различными методами незначительно по сравнению с расхождением, получаемым каждым из этих методом при использовании начальных данных, заданных в другие моменты оскуляции. Так как оба метода дают незначительные расхождения в элементах орбит, то это позволяет считать, что метод с использованием банка данных

Таблица 1

Оскулирующие элементы орбит короткопериодических комет $(T=21901226)$

\begin{tabular}{|c|c|c|c|c|c|c|}
\hline & $M$ & $a$ & $e$ & $\omega$ & $\Omega$ & $i$ \\
\hline \multicolumn{7}{|c|}{$\mathrm{P} /$ Halley } \\
\hline $\begin{array}{c}\text { Совм. интегр. } \\
\text { Б. дан. коэф. } \\
\quad \Delta S \\
\end{array}$ & $\begin{array}{c}273,2761 \\
273,2790 \\
0,0029 \\
\end{array}$ & $\begin{array}{c}17,74513 \\
17,7454 \\
0,00009 \\
\end{array}$ & $\begin{array}{c}0,967199 \\
0,967199 \\
0 \\
\end{array}$ & $\begin{array}{c}115,3018 \\
115,3018 \\
0 \\
\end{array}$ & $\begin{array}{c}62,6713 \\
62,6714 \\
0,0001 \\
\end{array}$ & $\begin{array}{c}161,6867 \\
161,6867 \\
0 \\
\end{array}$ \\
\hline \multicolumn{7}{|c|}{$\mathrm{P} /$ Encke } \\
\hline $\begin{array}{c}\text { Совм. интегр. } \\
\text { Б. дан. коэф. } \\
\Delta S \\
\end{array}$ & $\begin{array}{c}232,9695 \\
232,9664 \\
0,0001 \\
\end{array}$ & $\begin{array}{l}2,211109 \\
2,211110 \\
0,000001 \\
\end{array}$ & $\begin{array}{c}0,851064 \\
0,851064 \\
0 \\
\end{array}$ & $\begin{array}{c}191,5234 \\
191,5234 \\
0 \\
\end{array}$ & $\begin{array}{c}330,9684 \\
330,9684 \\
0 \\
\end{array}$ & $\begin{array}{c}9,6482 \\
9,6482 \\
0 \\
\end{array}$ \\
\hline \multicolumn{7}{|c|}{ D/Biela } \\
\hline $\begin{array}{c}\text { Совм. интегр. } \\
\text { Б. дан. коэф. } \\
\Delta S \\
\end{array}$ & $\begin{array}{c}113,4167 \\
113,4123 \\
0,0044 \\
\end{array}$ & $\begin{array}{l}3,521668 \\
3,521675 \\
0,000007 \\
\end{array}$ & $\begin{array}{l}0,764597 \\
0,764598 \\
0,000001 \\
\end{array}$ & $\begin{array}{c}323,0907 \\
323,0895 \\
0,0012 \\
\end{array}$ & $\begin{array}{c}143,3537 \\
143,3548 \\
0,0011 \\
\end{array}$ & $\begin{array}{c}13,8526 \\
13,8519 \\
0,0007 \\
\end{array}$ \\
\hline \multicolumn{7}{|c|}{$\mathrm{P} /$ Faye } \\
\hline $\begin{array}{c}\text { Совм. интегр. } \\
\text { Б. дан. коэф. } \\
\Delta S\end{array}$ & $\begin{array}{c}292,0967 \\
292,0967 \\
0 \\
\end{array}$ & $\begin{array}{c}3,798059 \\
3,798059 \\
0 \\
\end{array}$ & $\begin{array}{c}0,592634 \\
0,592634 \\
0 \\
\end{array}$ & $\begin{array}{c}217,8278 \\
217,8279 \\
0,0001 \\
\end{array}$ & $\begin{array}{c}176,4265 \\
176,4265 \\
0\end{array}$ & $\begin{array}{c}6,2071 \\
6,2071 \\
0 \\
\end{array}$ \\
\hline \multicolumn{7}{|c|}{$\mathrm{D} /$ Brorsen } \\
\hline $\begin{array}{c}\text { Совм. интегр. } \\
\text { Б. дан. коэф. } \\
\Delta S\end{array}$ & $\begin{array}{c}189,9286 \\
189,9282 \\
0,0004\end{array}$ & $\begin{array}{l}3,080769 \\
3,080765 \\
0,000004\end{array}$ & $\begin{array}{c}0,862202 \\
0,862202 \\
0\end{array}$ & $\begin{array}{c}73,1458 \\
73,1433 \\
0,0035\end{array}$ & $\begin{array}{c}39,9301 \\
39,9337 \\
0,0036\end{array}$ & $\begin{array}{l}6,7641 \\
6,7643 \\
0,0002\end{array}$ \\
\hline
\end{tabular}




\begin{tabular}{|c|c|c|c|c|c|c|}
\hline & $M$ & $a$ & $e$ & $\omega$ & $\Omega$ & $i$ \\
\hline \multicolumn{7}{|c|}{ Apollo / 2003 HP32 } \\
\hline Совм. интегр. & 295,2300 & 2,707196 & 0,7744689 & 173,9676 & 172,5070 & 7,1955 \\
\hline Б. дан. коэф. & 295,3061 & 2,707038 & 0,774676 & 173,9630 & 172,5108 & 7,1958 \\
\hline$\Delta S$ & 0,0761 & 0,000158 & 0,000013 & 0,0046 & 0,0038 & 0,0003 \\
\hline \multicolumn{7}{|c|}{ Aten / 2003 HT42 } \\
\hline Совм. интегр. & 266,8799 & 0,815117 & 0,258110 & 354,9099 & 33,8183 & 5,0272 \\
\hline Б. дан. коэф. & 266,8797 & 0,815117 & 0,258110 & 354,9099 & 33,8183 & 5,0272 \\
\hline$\Delta S$ & 0,0002 & 0 & 0 & 0 & 0 & 0 \\
\hline \multicolumn{7}{|c|}{ Apollo / 2005 VG7 } \\
\hline Совм. интегр. & 226,9027 & 1,993375 & 0,548335 & 329,9585 & 37,9509 & 2,8599 \\
\hline Б. дан. коэф. & 226,9023 & 1,993375 & 0,548335 & 329,9585 & 37,9509 & 2,8599 \\
\hline$\Delta S$ & 0,0004 & 0 & 0 & 0 & 0 & 0 \\
\hline \multicolumn{7}{|c|}{ Aten / 1998 SZ27 } \\
\hline Совм. интегр. & 174,4909 & 0,903783 & 0,504279 & 48,3157 & 165,9540 & 23,3899 \\
\hline Б. дан. коэф. & 174,4905 & 0,903783 & 0,504279 & 48,3156 & 165,9540 & 23,3899 \\
\hline$\Delta S$ & 0,0004 & 0 & 0 & 0,0001 & 0 & 0 \\
\hline \multicolumn{7}{|c|}{ Aten / $1994 \mathrm{GL}$} \\
\hline Совм. интегр. & 182,2678 & 0,683916 & 0,504812 & 187,0201 & 189,1222 & 3,7599 \\
\hline Б. дан. коэф. & 182,2596 & 0,683917 & 0,504811 & 187,0213 & 189,1213 & 3,7607 \\
\hline$\Delta S$ & 0,0084 & 0,000001 & 0,000001 & 0,0012 & 0,0009 & 0,0008 \\
\hline
\end{tabular}

координат, скоростей, ускорений и коэффициентов $A_{1}, A_{2}, A_{3}, A_{4}$ больших планет, Луны и Солнца можно применять как для исследования эволюции орбит короткопериодических комет, так и для астероидов групп Аполлона, Амура, Атона.

Работа выполнена при поддержке Министерства образования и науки РФ (проект РНП 2.1.1/14069).

\section{БИБЛИОГРАФИЧЕСКИЙ СПИСОК}

1. Заусаев А.Ф., Заусаев Д.А. Численное интегрирование уравнений движения малых тел солнечной системы с использованием оскулирующих элементов больших планет / В сб.: Труды шестой Всероссийской научной конферениии с международным участием (1-4 июня 2009 г.). Часть 3: Дифференциальные уравнения и краевые задачи / Матем. моделирование и краев. задачи. Самара: СамГТУ, 2009. С. 125-130. [Zausaev A. F., Zausaev A. A. Numerical integration of the equations of motion of small solar system bodies with using the osculating elements for major planets / In: Proceedings of the Sixth All-Russian Scientific Conference with international participation (1-4 June 2009). Part 3 / Matem. Mod. Kraev. Zadachi. Samara: Samara State Technical Univ., 2009. Pp. 125-130].

2. Newhall X.X., Standish E.M., Williams J. G. DE 102: A numerically integrated ephemeris of the moon and planets spanning forty-four centuries // Astron. Astrophys., 1983. Vol. 125, no. 1. Pp. 150-167.

3. Standish E. M. JPL Planetary and Lunar Ephemerides, DE405/LE405: Jet Prop Lab Technical Report. IOM 312. F-98-048, http://iau-comm4.jpl.nasa.gov/de405iom/ de405iom.pdf.

4. Заусаев А. Ф, Заусаев А. А. Каталог орбитальной эволюции короткопериодических комет с 1800 по 2204 гг. М.: Машиностроение-1, 2007. 410 с. [Zausaev A.F., Zausaev A. A. Catalogue of the orbital evolution of short-period comets (1800-2204). Moscow: Mashinostroenie-1, 2007. $410 \mathrm{pp}$.]

5. Заусаев А.Ф., Абрамов В.В., Денисов С. С. Каталог орбитальной эволюции астерои- 
дов, сближающихся с Землей с 1800 по 2204 гг. М.: Машиностроение-1, 2007. 608 с. [Zausaev A. F.,Abramov V. V., Denisov S.S. Catalogue of the orbital evolution of near-Earth asteroids (1800-2204). Moscow: Mashinostroenie-1, 2007. 608 pp.]

Поступила в редакцию $28 / \mathrm{II} / 2011$

в окончательном варианте - 12/IX/2011.

MSC: 85-08; 70M20, 65L99

\section{USING THE DATABASE OF COORDINATES OF THE MAJOR PLANETS FOR THE NUMERICAL INTEGRATION OF THE EQUATIONS OF CELESTIAL BODIES MOTION}

\section{V.P. Radchenko, D. A. Zausaev}

Samara State Technical University,

244, Molodogvardeyskaya st., Samara, 443100, Russia.

E-mails: radch@samgtu.ru, zadmitriy@gmail.com

Databases of major planets coordinates are created on the basis of the numerical theory of movement of planets DE405. Numerical integration of the motion equations of small bodies of Solar system is realized using the received databanks. Extra efficiency of usage of databanks of major planets coordinates is shown at numerical integration of motion equations of celestial bodies.

Key words: interpolation, asteroids, comets, numerical integration.

Original article submitted 28/II/2011; revision submitted 12/IX/2011.

Vladimir P. Radchenko (Dr. Sci. (Phys. \& Math.)), Head of Dept, Dept. of Applied Mathematics \& Computer Science. Dmitriy A. Zausaev, Postgraduate Student, Dept. of Applied Mathematics \& Computer Sciences. 